\title{
Expectativas y valoraciones de futuros maestros sobre innovaciones didácticas
}

Expectations and assessments of future teachers on didactic innovations

\author{
Mónica Porto Currás, Mª José Bolarín Martínez, Bárbara Iborra Álvarez \\ Departamento de Didáctica y Organización Escolar \\ Universidad de Murcia \\ Campus Espinardo, 30100, Murcia \\ monicapc@um.es mbolarin@um.es babi_iborra@hotmail.com
}

\begin{abstract}
Resumen
Este artículo tiene como objetivo conocer las expectativas y valoraciones de futuros maestros respecto a las innovaciones que emplean sus docentes; objetivo enmarcado en un proyecto de investigación internacional, financiado por la Secretaría de Ciencia y Técnica y Estudios Regionales de la Universidad Nacional de Jujuy y desarrollado entre los años 2012 y $2015^{1}$.

Con una metodología cualitativa y longitudinal, los datos se obtuvieron de una muestra de estudiantes del Grado de Maestro al inicio y en el último mes de su formación universitaria. Los principales resultados apuntan, aunque con matices, a que: 1) la forma en que los profesores enseñan condiciona la metodología de los futuros docentes; 2) las innovaciones tecnológicas han sido más relevantes que las metodológicas y 3) las características que muestra el docente en la creación de ambientes de aprendizaje se encuentran entre los aspectos destacados como necesarios para mejorar las experiencias formativas del alumnado.
\end{abstract}

Palabras clave: Expectativas del estudiante, Formación de profesores, Innovaciones educativas, Investigación cualitativa, Voz del alumnado.

\begin{abstract}
This article aims to know the expectations and assessments of future teachers about the innovations used by their professors; objective of an international research project, funded by the Secretariat for Science and Technology and Regional Studies at the National University of Jujuy and developed between 2012 and 2015.

Based on a qualitative and longitudinal methodology, the data were obtained from a sample of students from the Teacher Degree at the start and in the last month of their university training. The main results point, although with nuances, to: 1) the manner in which teachers teach determines the methodology of future teachers; 2) technological innovations have been more relevant than methodological and 3) how teachers create learning environments are among the outstanding aspects and needed for improving the educational experiences of students.
\end{abstract}

Key words: Educational innovations, Qualitative research, Student expectation, Teacher education, Voice of pupils.

\footnotetext{
${ }^{1}$ Expectativas de jóvenes estudiantes de grupos heterogéneos respecto a las innovaciones de sus profesores/capacitadores para mejorar los aprendizajes e insertarse laboralmente. Proyecto de investigación. Código SECTER - UNJU - D.23.5, Dirigido por la Dra. Rosa Pérez del Viso de Paloy y la Dra. Beatriz Guerci de Siufi.
} 


\section{Antecedentes}

Con frecuencia se denuncia una carencia de procesos de innovación en las instituciones educativas y, especialmente, en las universidades, que provoca en los alumnos una falta de respuesta creativa y eficaz que les permita enfrentarse a las necesidades de la sociedad actual. Algunos docentes opinan que los estudios superiores, al haber sido elegidos por los alumnos, deben suponer una motivación que les permita aprender sin necesidad de preocuparse por modificar su metodología docente. Es evidente que los estudios post secundarios no constituyen un tramo obligatorio y, si el alumno ha elegido esa carrera, cabría suponer una gran motivación por aprovechar al máximo sus estudios, pero en realidad, la motivación no es propiedad ni responsabilidad exclusiva del alumno. Más aún, el estudiante se siente proclive a depositar en el docente las responsabilidades de su aprendizaje. Desde esta óptica, cada vez son más los que piensan que su condición de docente conlleva crear contextos que permitan hacer las clases más interesantes y que den respuesta a las necesidades de sus estudiantes. Sin embargo, también es frecuente la falta de coincidencias entre las expectativas del alumnado y las innovaciones docentes que se efectúan (Clark, 2011).

En algunas investigaciones previas (Pérez del Viso, 2010) se ha observado como mayoritariamente los estudiantes no se plantean la repercusión de las innovaciones llevadas a cabo por sus profesores en sus resultados académicos y en su futuro laboral. Sin embargo, las aulas funcionan de forma sistémica, influenciándose todos los actores entre sí y con el contexto y, por lo tanto, al modificar las partes o su funcionamiento, se deberían modificar los resultados de los aprendizajes y competencias profesionales fomentados. O'Connort y Mc Dermott (2008: pp. 28-29) sostienen que en la educación se ha descuidado el análisis del pensamiento sistémico que da cuenta de que el "(...) comportamiento depende de la estructura global. Si se cambia la estructura se modifica el comportamiento del sistema". Las aulas también funcionan de forma sistémica, por lo que no se pueden analizar sus partes de forma inconexa, cada modificación o innovación que se realiza, por muy ligera que sea, influye en el funcionamiento conjunto de la clase.

Es evidente que el que puede innovar la organización es básicamente el profesor. Sin embargo, cuando los alumnos más avanzados han logrado un pensamiento crítico, señalan cuales son las experiencias de enseñanza que han provocado en ellos mejores aprendizajes. Consecuentemente, advertimos como no son sólo importantes los contenidos desarrollados durante los estudios, sino también los métodos que se desarrollan para enseñarlos y las expectativas de los discentes sobre ellos. Tal y como se plantea en la fundamentación del proyecto de investigación: la investigación educacional plantea en las últimas décadas algunas reconceptualizaciones a considerar, con tendencia a asumir una perspectiva orientadora y multidimensional que afirma que la cognición y la motivación son inseparables para la comprensión de cualquier comportamiento humano. Se enfatiza la naturaleza social y situada del aprendizaje académico, buscando describir cómo las creencias y motivaciones de los estudiantes interactúan con las señales presentes en los diversos ambientes instruccionales, físicos y sociales, resultando el contexto un coconstructor del proceso motivacional, ya que lo que cada uno hace y aprende influye en las tareas subsecuentes. Cuanto mayor es la eficacia percibida por el alumno en su actuar, más complejas son las metas que se propone para sí y hacia sus profesores, sintiéndose con el derecho de cuestionar los trabajos e instrumentos didácticos ofrecidos por éstos.

Entendemos, por tanto, que enseñanza y aprendizaje son actividades concomitantes. En este sentido, Trillo (2008) manifiesta que entre el concepto de enseñanza-aprendizaje hay una relación ontológica, es decir, no se puede entender una sin el otro y viceversa. Si no se produce el proceso de aprendizaje adecuado hay que revisar el proceso de enseñanza y, para que se produzca aprendizaje, siempre ha de haber un estímulo de partida, una experiencia, una acción, un contexto de enseñanza (más o menos reglado, más autónomo o más dependiente, etc.). En segundo lugar, al hablar de enseñanza, se habla tanto de una actividad encaminada a lograr un aprendizaje como de una intencionalidad por parte del profesor. Esta intencionalidad es tanto del profesor, que enseña, como del alumno, que aprende, y podemos decir, más concretamente que, en el caso del alumnado depende de múltiples factores, entre los que cabe destacar 
aquellos factores personales que, además de las cualidades propias personales de carácter intelectual, conductual y emocional, hay que tener en cuenta la presencia de expectativas, atribuciones, hábitos, prejuicios, conocimientos y habilidades, actitudes, valores, etc., que se desarrollan a lo largo de la historia de vida de los estudiantes, motivación, conocimientos previos, etc. Desde esta perspectiva, "es necesario contemplar la atmósfera creada por el profesor en el aula, la forma en que interactúa con los alumnos y el ambiente físico en que se desarrolla" (Muijs y Reynolds, 2002, p.107) en aras de una enseñanza de calidad. Esto significa que el comportamiento docente en relación con los alumnos es de fundamental importancia, para que ocurra el aprendizaje (Lafout, 1999).

El profesor se convierte así en parte fundamental del proceso enseñanza-aprendizaje y, su intención básica es crear las condiciones necesarias para que sus alumnos aprendan. A pesar de que, según Hattie (2003) y Rowe (2004), una enseñanza eficaz depende principalmente de los alumnos (50\%) dejando un porcentaje del $30 \%$ a maestros y un $20 \%$ a la familia, compañeros e institución educativa, son los profesores los responsables de establecer un entorno que motive a los alumnos en el proceso de enseñanza- aprendizaje.

La profesionalidad del docente no se reduce solamente a una selección y exposición de los contenidos en clase, sino que debe ser un profesional capaz de reflexionar a partir de su práctica y ser capaz de actuar de manera eficaz en un contexto concreto, de innovar para adaptarse a las necesidades del alumnado. Desde esta perspectiva, el profesor es el encargado de generar en los alumnos la necesidad de aprender, debe ser un agente activo, capaz de conseguir que el alumnado desarrolle un interés por aprender. Si además estamos hablando de alumnos del Grado de Magisterio, estas innovaciones docentes tienen que permitirle al alumnado mejorar su capacitación profesional, darle conocimientos y recursos para poder desarrollar también estas competencias en su futuro docente.

Así, como señalan Pintrich y Schunk (2006, citado en Bono, 2010) los profesores influyen en la motivación y el aprendizaje de sus estudiantes a través de su planificación e instrucción, y, a su vez, la manera en que los estudiantes reaccionan provoca en los profesores modificaciones que, ellos entienden, afectarán de mejor manera la motivación y el aprendizaje.

\section{Metodología}

Como se ha anticipado, en este observatorio pretendíamos aproximarnos a las representaciones sociales y expectativas de una muestra de estudiantes del Grado de Maestro en Educación Primaria (63 estudiantes) sobre las posibilidades de alcanzar aprendizajes aptos o bien de incrementar sus aprendizajes y posibilidades laborales según las innovaciones que sus profesores universitarios implementan. Para ello, se realizó una encuesta al inicio de su formación docente, en el primer cuatrimestre de su primer curso universitario y otra encuesta a la misma muestra de estudiantes en el último cuatrimestre del último curso de su formación universitaria de Grado. Se persigue así conocer sus expectativas iniciales en relación a las innovaciones docentes que iban a vivir como futuros maestros y su relación con su formación profesional, así como la valoración de las innovaciones docentes (si las hubo) al finalizar esta formación inicial.

La metodología de investigación empleada fue de corte cualitativo, estudio descriptivo-exploratorio (Ruiz, 2012), utilizando como instrumento el cuestionario abierto. En él se plantean unos interrogantes a los estudiantes de un grupo del Grado de Maestro en Educación Primaria de la Universidad de Murcia (promoción 2011-2015) con el objeto de que expresen libremente sus ideas, creencias, opiniones, valoraciones (Tójar, 2006); y, por tanto, considerándoles colaboradores imprescindibles en nuestra investigación.

A partir de las respuestas obtenidas, el proceso de análisis se centró en "dar sentido" a la información textual. Para ello, se utilizó la base de datos Microsoft Excel, que permitió introducir el conjunto de respuestas obtenidas, ofreciendo una visión general de toda la información y facilitando la codificación de las respuestas y el establecimiento de categorías. De este modo, la información proporcionada fue 
categorizada atendiendo a criterios temáticos (Rodríguez, Gil y García, 1996), a partir del análisis del contenido de las aportaciones obtenidas y siguiendo la vía inductiva, a medida que se va analizando el texto.

La encuesta se centró en primer curso (octubre 2011) en las siguientes cuestiones: relación entre la forma de ejercer la docencia de los docentes y su formación como maestros; las innovaciones que esperan encontrar en sus estudios universitarios y las que les gustaría; y sus creencias sobre cómo deberían enseñar sus docentes para mejorar su formación como futuros maestros de Educación Primaria.

Al finalizar su formación inicial, último cuatrimestre del cuarto curso de grado (mayo 2015), se realizaron las siguientes preguntas al mismo grupo de estudiantes: ¿Qué innovaciones docentes has encontrado a lo largo de esta carrera?; ¿cómo crees que se relaciona la forma de enseñar de tus profesores con tu formación profesional?; ¿cómo debería haber sido la enseñanza para mejorar tu formación como docente de Educación Primaria?

Debe matizarse el hecho de que el número de alumnos que respondieron la encuesta en primer y último cuatrimestre de su formación coincide totalmente, siendo el número total de estudiantes asistentes y que continuaron en la carrera hasta el final en ese grupo (uno de los siete grupos del Grado de Maestro en Educación Primaria que se ofrecen en la Universidad de Murcia) y que constituye el 93\% de los alumnos matriculados en primero.

\section{Resultados}

Las aportaciones de los estudiantes de primer curso se organizaron en las categorías que se muestran en la Tabla 1.

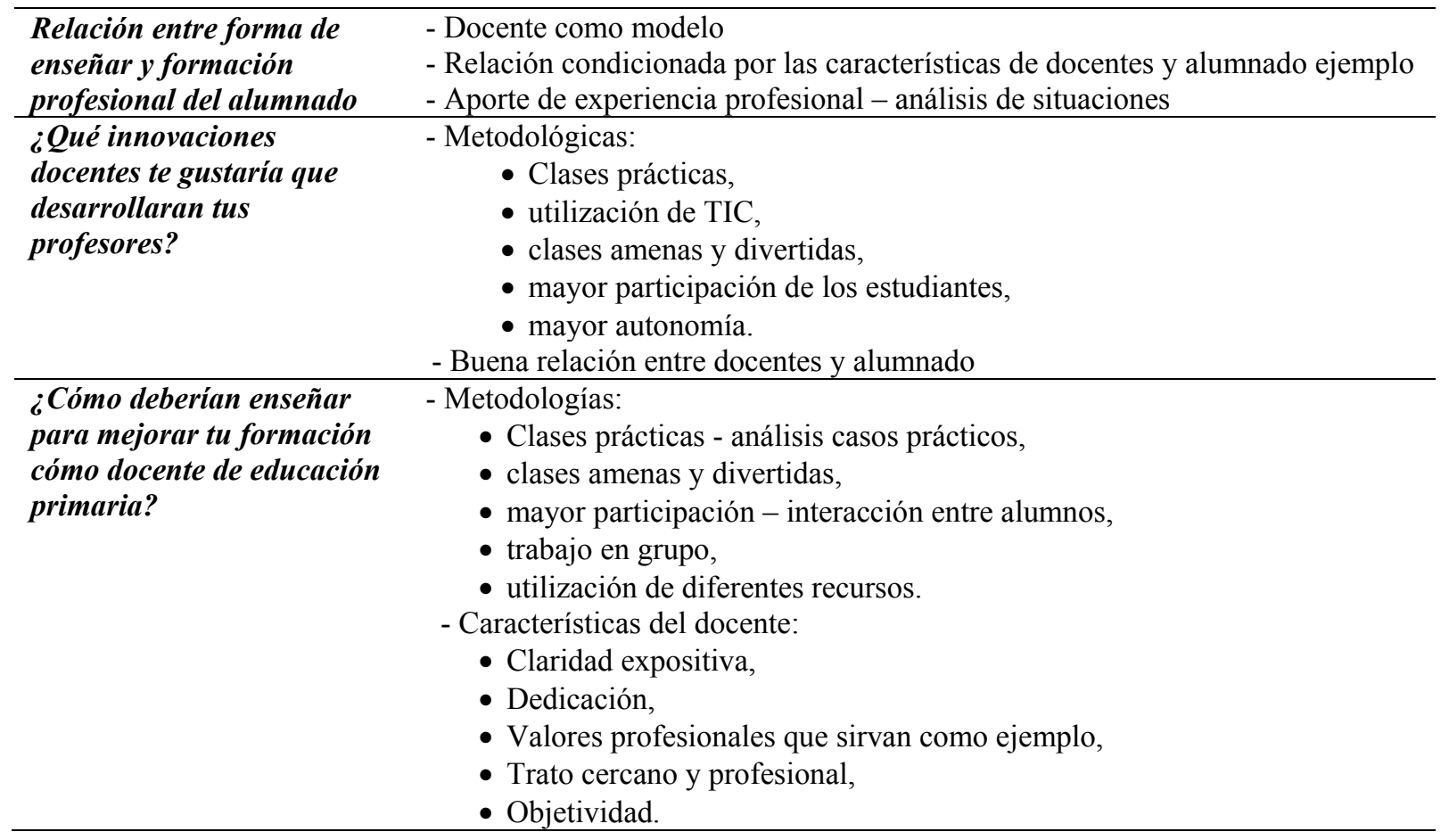

Tabla 1: Categorización de las aportaciones del alumnado de $1^{\mathrm{o}}$

Mostramos a continuación un análisis de las respuestas dadas en cada categoría. 


\section{Relación entre la forma de enseñar del docente y la formación profesional del alumnado según el alumnado de primer curso}

La mayoría de los alumnos encuestados (29 de 63), consideran que la forma en que sus profesores enseñan será un modelo o ejemplo que tendrán para ejercer su futura profesión. Reconocen, además, que estos modelos no siempre se adoptan de forma consciente, sino que, tal y como está recogido en la bibliografía especializada, con frecuencia es de forma inconsciente como se sigue el sistema metodológico que se ha vivido como alumnos: Creo que están directamente relacionadas. Es decir, en numerosas ocasiones los alumnos adoptamos inconscientemente o incluso conscientemente metodologías, sistemas, formas de expresión y hasta energías de nuestros profesores (A13); Creo que en un futuro la labor que van a desarrollar mis docentes repercutirá directamente en mi forma de enseñar, en mi visión sobre como hay que educar y en general en mi forma de ser profesionalmente (A25).

Precisamente, cuando hablan de qué aspectos son los que les pueden servir como ejemplo de sus profesores, los estudiantes de nuestra muestra se centran tanto en aspectos metodológicos, contenidos que enseñan explícitamente, como en cuestiones más relacionadas con valores profesionales (responsabilidad, cuidado de los alumnos) o incluso personales de sus docentes (energía, entusiasmo).

No obstante, algunos alumnos consideran que la influencia ejercida no depende sólo de la forma de enseñar de sus docentes, sino también de la personalidad del alumno y claridad que tenga sobre la docencia que le gustaría desarrollar en un futuro. Así, un número considerable de estudiantes (14 de 63) responden a la cuestión planteada afirmando que la influencia que puede haber entre la forma de enseñar de sus docentes y su formación profesional viene condicionada por diferentes características de profesores y alumnos, así como el clima que se cree entre ambos. Entre las características que señalan podemos destacar: implicación, interés y preparación de ambos sujetos; adaptación al nivel de formación del alumnado; alta preparación del docente; presentación de los contenidos de forma clara y amena; selección de contenidos útiles.

En tercer lugar, encontramos las respuestas de algunos estudiantes ( 8 de 63) que consideran que en su formación profesional influyen especialmente las situaciones reales que pueden mostrarle los docentes universitarios, así como las experiencias que estos docentes hayan tenido para enseñar en esas situaciones. Partiendo de esas experiencias podrán ejemplificar lo que están explicando y ayudarles a entender cómo se aplican los conocimientos teóricos a las situaciones que, como futuros docentes, se enfrentarán.

\section{¿Qué innovaciones docentes esperas encontrar a lo largo de esta carrera? ¿Cuáles te gustaría que desarrollaran tus profesores?}

La mayoría de las declaraciones de los alumnos encuestados (51 de 63) expresan que, fundamentalmente, lo que esperan en la formación como docentes es un mayor uso de clases prácticas que las que han tenido en los niveles educativos anteriores: Las clases prácticas, ya que en la E.S.O. es algo que no solemos tener, y dar este tipo de clases puede ser una innovación, además de la forma de exponer las clases (A21).

Además, en sus declaraciones muestran ejemplos de a qué se refieren con clases prácticas o el tipo de actividades que les gustaría que se desarrollaran en ella: trabajos en grupo, análisis de situaciones reales que les permitan conocer y aprender a solucionar las situaciones que puedan aparecer en su futuro profesional, debates que permitan hacer explícitas las diferentes opiniones, perspectivas o formas de entender los contenidos de los estudiantes y trabajos de investigación.

Por otra parte, un número importante de declaraciones de los estudiantes (26 de 63) hacen referencia a la utilización de tecnologías como la innovación que esperan encontrar en sus estudios del Grado de Maestro. Entre estas innovaciones tecnológicas, 8 estudiantes se refieren al uso de Tecnologías para hacer las presentaciones de los contenidos más claras y otros 4 aluden al uso del aula virtual como espacio de comunicación entre docentes y alumnado. 
En otro sentido, 10 de las declaraciones de estos estudiantes aluden a su expectativa que las clases que se encuentren en el Grado de Maestro de Educación Primaria sean clases amenas y divertidas: Además de las anteriores me gustaría que mis profesores hiciesen más amenas las clases (A18); Espero que hagan las clases amenas. Además me gustaría que los profesores realizasen juegos y métodos divertidos para enseñar (A51).

Como complemento a la categoría anterior, 12 respuestas de los 63 estudiantes de esta muestra aluden a que esperan que en esta nueva etapa de su formación se cuente con una mayor participación del alumnado, fomentando su implicación y teniendo en cuenta sus sugerencias y aportaciones para desarrollar las sesiones formativas.

Otras 6 respuestas de estos estudiantes hacen referencia a su expectativa de poder desarrollar un trabajo más autónomo, de poder ir mejorando su capacidad para trabajar de forma autónoma progresivamente: Espero encontrar más autonomía a la hora de trabajar y estudiar, es decir, que seamos más capaces de llegar a nuestros objetivos por nosotros mismos (A45).

Por último, aunque no siempre lo presentan como una innovación, 16 de las 63 respuestas obtenidas hacen referencia a la necesidad de una buena relación entre docentes y alumnado en su formación. De esta forma, utilizan concepciones como trato cercano, respeto, comprensión, ayuda y mayor interacción entre profesor y estudiante para referirse a la relación que esperan que se desarrolle en la acción educativa.

\section{¿Cómo deberían enseñar para mejorar tu formación cómo docente de educación primaria?}

La última cuestión que le planteamos a los estudiantes de primer curso intentaba aproximarnos a sus creencias de cómo sería una docencia que repercuta positivamente en su formación profesional como maestros de Educación Primaria. Sus respuestas nos ofrecen categorías muy similares a las halladas en la cuestión anterior, reiterando sus preferencias por el empleo de metodologías didácticas centradas en clases prácticas donde se analicen situaciones reales, estructuras de trabajo cooperativas, mayor interacción entre docentes y alumnos, y todo ello con un docente que se caracterice por su dedicación, claridad expositiva y trato cercano con los alumnos.

Y, por último, debemos hacer referencia a dos declaraciones de estudiantes que consideran que las innovaciones que se introduzcan no debieran obviar aquello que funcionaba bien de los métodos tradicionales, de forma que también les gustaría que se mantuvieran algunas de las características de sus docentes anteriores.

Por su parte, las aportaciones de los estudiantes de cuarto curso se organizaron en las categorías que se muestran en la Tabla 2.

\begin{tabular}{ll}
\hline ¿Cómo crees que se relaciona la forma de & - Relación directa \\
enseñar de tus profesores con tu formación & - Relación negativa, falta de coherencia \\
profesional? & - Relación variable \\
& - No se relacionan \\
\hline & - Tecnológicas \\
¿Qué innovaciones docentes (con respecto & - Metodologías: \\
a tus experiencias en Educación & - Trabajo en grupo \\
Secundaria) has encontrado a lo largo de & - Clases prácticas \\
esta carrera? & - Casos reales \\
Utilidad de estas innovaciones & - Conocimiento de metodologías / estrategias innovadoras \\
& - Participación activa \\
& - Trabajo cooperativo \\
& - Exposiciones de alumnado \\
\hline
\end{tabular}




\begin{tabular}{ll}
\hline & $\bullet$ Charlas de expertos \\
& - Otros (role-playing, investigaciones, ...) \\
& - Ninguna innovación \\
\hline ¿Cómo debería haber sido la enseñanza & - Metodologías: \\
para mejorar tu formación como docente & - Enseñanza más práctica \\
de Educación Primaria? & - Enseñanza más activa y dinámica \\
& - Contenidos específicos \\
& - Prácticas Escolares Externas \\
& - Mayor dedicación y motivación docente \\
\hline
\end{tabular}

Tabla 2: Categorización de las aportaciones del alumnado de $4^{\circ}$

Al igual que en el caso anterior, desarrollamos a continuación estas categorías con más detalle:

\section{¿Cómo crees que se relaciona la forma de enseñar de tus profesores con tu formación profesional?}

La mayor parte de las respuestas declaradas a este interrogante (19 de 63) consideran que los docentes que han tenido a lo largo de la carrera de maestros, su forma de impartir docencia, influirá notablemente en su formación profesional: Creo que se relaciona directamente porque los profesores siguen siendo el "modelo" a imitar y aunque después se actúe de forma crítica y personal siempre influye su forma de dar clase y sus enseñanzas (A07).

No obstante, casi el mismo número de estudiantes (18 de 63) aluden a una relación negativa, es decir, creen que sus profesores han sido un ejemplo negativo para su formación como docentes: Negativamente pues en la mayoría de los casos, los profesores han representado todo lo contrario al modelo que espero seguir en mi futuro, tanto metodológicamente, como personalmente (A01).

En esta categoría se incluyen también declaraciones de estudiantes (10 de 63) que consideran que entre sus docentes han encontrado tanto como ejemplos a imitar como, en algunos casos, errores que no quieren reproducir: Dicha relación depende del profesor que haya impartido las distintas asignaturas. Algunos son un modelo a seguir, tanto por su trato con el alumnado, como por la metodología usada para transmitir conocimiento, sin embargo otros son modelo de aquellos que nunca deberé hacer como docente (A02).

Y, por último, encontramos las declaraciones de 5 estudiantes que consideran que no hay relación entre la forma de enseñar de sus docentes y su formación profesional, aludiendo, principalmente, a la poca adecuación entre los contenidos impartidos y los que ellos como maestros podrán enseñar en Educación Primaria: Creo que en esta carrera, la mayoría de profesores se dedican a impartir conocimientos que no tienen relación con nuestra profesión, los contenidos no se adecuan a lo que en un futuro enseñaremos sobre todo los tres primeros años de universidad. En resumen, no nos enseñan a ser buenos docentes (A60).

\section{¿Qué innovaciones docentes (con respecto a tus experiencias en Educación Secundaria) has encontrado a lo largo de esta carrera?}

La mayor parte de los estudiantes aluden a innovaciones tecnológicas como la principal innovación que han encontrado en los estudios de Magisterio: Los más útiles para mi formación como maestro han sido las que me han presentado ejemplos de intertextualidad (A22).

Entre estas innovaciones tecnológicas podemos destacar, de acuerdo con sus afirmaciones:

- Presentaciones visuales (20 de 63) 
- Aula Virtual (13 de 63)

- Pizarra digital o proyector 6 de 63)

- Vídeos (5 de 63)

- Blogs o páginas web (3 de 63)

- Tutorías electrónicas (3 de 63)

- Herramientas de Internet (que desconocían y afirman que les facilita mayor accesibilidad a la información, 2 de 63)

Las otras innovaciones reconocidas por los estudiantes de esta muestra se refieren a innovaciones metodológicas (38 de 63), que se han organizado en los siguientes epígrafes:

- Trabajos en grupo: Respecto a la forma de dar las clases se favorece el trabajo grupal, lo que propicia una mayor cooperación entre los alumnos, a diferencia de las clases de la ESO, que solo hace trabajos individuales (A37).

- Prácticas: Ahora se realizan actividades prácticas que permiten un aprendizaje más significativo (A07), Actividades de manipulación y experimentación (A41).

- Conocimiento de diversas metodologías / estrategias innovadoras: Trabajos por proyectos, trabajo por tareas, ABP, inclusión de las TIC, etc. (A36); Otra podría ser el visionado de diversas metodologías de enseñanza (aprendizaje cooperativo, tutorías entre iguales, clases magistrales...) (A46).

- Participación activa: A nivel metodológico si ha habido docentes que se han salido de la enseñanza tradicional basada en el libro de texto y han optado por proporcionar un papel activo al alumno, que él fuera constructor de su aprendizaje (A02); La participación activa del alumnado en clase (A27).

- Casos reales: Análisis de casos reales (A14).

- Charlas por parte de expertos: Un par de charlas que nos han dado expertos en temas importantes para nuestra formación, en las horas de clase (A48).

- Trabajo cooperativo: El trabajo cooperativo, aunque muchas veces con una gestión deficiente (A47).

- Realización de exposiciones: Realizamos muchas exposiciones orales, que permiten que nos soltemos (A59).

- Otros: Entre las que se aluden a metodologías o estrategias metodológicas como: Role playing, clases dinámicas, realización de diarios de asignaturas, Investigación (La mejor estrategia es la que han utilizado un par de profesores y consiste en hacer que los alumnos investiguemos sobre un tema en base a los documentos que nos ofrecen y en grupos, presentan la información a nuestros compañeros. De esta forma, no es siempre la profesora quien imparta los conocimientos (A58); experiencias en laboratorio; críticas de lecturas, etc.

Por último, no podemos obviar que un número considerable de estudiantes (12 de 63) consideran que no ha habido ninguna innovación en la forma de enseñar de sus docentes de Magisterio que pueda haber influido en su formación profesional como futuros maestros: Sinceramente, no considero que a lo largo de la carrera haya habido ninguna innovación, ya que las clases (al igual que en Educación Secundaria) se han basado en la lección magistral, simplemente con ayuda de las TIC, pero en mi opinión eso no son innovaciones ya que la metodología sigue siendo la misma. Este último año hemos tenido otra asignatura de TIC, la cual no me ha gustado nada, ya que han "querido" trabajar con las TIC y la metodología ha sido igual (A08).

Para poder matizar las respuestas a esta pregunta, les pedimos a los estudiantes que nos dijeran también cuáles de esas innovaciones consideran que han sido más útiles para su formación como maestro/a. De los 63 alumnos solo 24 alumnos han respondido a esta pregunta y se han sintetizado sus aportaciones en las siguientes ideas:

- Las innovaciones tecnológicas han sido útiles en su mayoría, facilitando el contacto con los docentes, el acceso a la información o el conocimiento de nuevas herramientas digitales que permiten metodologías más dinámicas y atractivas. 
- Diferentes estrategias y metodologías didácticas, que han permitido un papel más activo y participativo del alumnado, como es el caso del trabajo por proyectos, metodologías basadas en la resolución de problemas, planteamiento de trabajos de investigación a realizar por los estudiantes, etc.

- No obstante, un reducido número de estudiantes (6 de los 24 que han respondido a esta pregunta), consideran que las innovaciones empleadas no han sido útiles para su formación.

\section{¿Cómo debería haber sido la enseñanza para mejorar tu formación como docente de Educación Primaria?}

Finalmente, cuestionamos al alumnado de último curso de su formación en el Grado de Maestros que nos plantearan como creen que debería haber sido la enseñanza recibida para mejorar su formación como docentes.

Sus respuestas se han categorizado en las siguientes dimensiones:

a) Enseñanza más práctica. Más de la mitad de los estudiantes (38 de 63) de esta muestra de estudiantes de Magisterio consideran que habría sido necesaria una enseñanza "más práctica", lo cual, de acuerdo con sus propias explicaciones, implicaría una enseñanza con mayor aplicación a las situaciones que se van a encontrar en una clase de Educación Primaria: Considero que mucho más práctico, pudiendo emplear todos los conceptos teóricos dados, haciendo todo más manipulativo, permitiendo ver los resultados en supuestos más similares a la realidad no solo en las prácticas (A04).

b) Más didáctica, recursos y estrategias. En segundo lugar, un tercio de los estudiantes (21 de 63) consideran que deberían haber podido vivenciar la utilización de mayor variedad de recursos y estrategias metodológicas en su formación, que les hubieran podido servir como ejemplo para emplear en su futuro profesional como docentes: Que me hubieran enseñado más recursos, más estrategias para poder enfrentarme a las clases de primaria con más confianza y experiencia (A16).

c) Enseñanza más activa y dinámica. Un tercer núcleo de respuestas (11 de 63) aluden a la necesidad de haber implicado más al alumnado, dejándole la oportunidad de tener un papel más activo en la construcción de sus aprendizajes, con mayor participación en el proceso de enseñanza: Tendría que ser más dinámica y más participativa (A33).

d) Contenidos específicos. Un reducido número de respuestas (5 de 63), insisten en la necesidad de que los contenidos que se abordan en su formación sean contenidos relacionados con los que ellos deben impartir en las clases de Educación Primaria: Reducir los contenidos de "relleno" y centrarse y profundizar en lo importante, cosas que se puedan explicar en clase con los niños (A40).

e) Prácticas escolares externas. También de forma reducida (4 de 63 estudiantes) se considera que las prácticas escolares externas que se realizan en centros de Educación Primaria deberían tener una duración mayor, para complementar y aplicar lo que aprenden en la Universidad: Más prácticas donde poder observar y trabajar la realidad educativa (A52).

f) Mayor dedicación y motivación docente. Por último, 3 de los 63 estudiantes encuestados consideran que faltaba mayor implicación y vocación por parte de su profesorado, cuestiones que serían necesarias para una docencia de mayor calidad formativa: Desde mi punto de vista, faltaba motivación e implicación en la mayoría de los casos. En gran medida, los profesores parecía que no tuviesen ganas de estar aquí, simplemente solo se preocupaban de llegar, "contar" el temario y ponernos una nota a partir de un examen teórico, lo que no me parece adecuado (A01).

\section{Conclusiones}

Los estudiantes de la muestra de $1^{\circ}$ del Grado de Maestro en Educación Primaria, de la Universidad de Murcia, creen que la forma en que sus profesores les enseñen determinará su futuro como docentes, ya que no solo les aportan conocimientos sobre contenidos y metodologías docentes, sino también las formas de ser y ejercer esta profesión. 
Desde esta perspectiva, consideran innovaciones todo aquello que no han vivido en su etapa de escolarización secundaria. Y cuando les pedimos que especifiquen que innovaciones creen que serían necesarias para mejorar su formación profesional sus respuestas inciden siempre en las mismas cuestiones:

- Metodologías más participativas, donde el papel del alumnado deje de ser el del receptor pasivo de información para tener que participar e debates, investigaciones, trabajos cooperativos... y donde se puedan analizar problemas reales. Estas declaraciones son coherentes con las exigencias de fomentar en el alumnado el desarrollo de las capacidades que le permitan hacer frente a las situaciones imprevistas y tomar iniciativas, responder de manera pertinente y adecuada, ser responsable y autónomo, movilizar recursos; relacionarse y comunicarse efectivamente con los demás, aceptar trabajar conjuntamente con un objetivo común, evaluar los efectos de las propias decisiones; perspectiva desde la que se reclama a la docencia universitaria que sea capaz de orientar, guiar y acompañar la formación de los estudiantes desde un saber de afirmaciones (lo sabido) a un pensar problematizador, creativo, como competencia clave para la búsqueda de respuestas apropiadas a los problemas actuales (Siufi, 2008).

- Utilización de todo tipo de medios didácticos, tecnológicos y materiales diversos que permitan el uso inteligente e imaginativo de metodologías, espacios y sistemas de evaluación para que aprender tenga sentido para los alumnos, de forma que se pueda revisar, valorar y cambiar cuanto sea preciso.

- Relación cercana entre docentes y alumnos, destacando los valores profesionales que deben caracterizar a los profesores. No se debería olvidar que las emociones son esenciales para el aprendizaje. Se exige desde esta perspectiva un ambiente de aprendizaje sensible a las diferencias entre el alumnado, que parta de su conocimiento previo y que no descuide las interacciones y relaciones pedagógicas entre y con los estudiantes.

Obviamente, estas cuestiones se complican cuando el número de alumnos por grupo es elevado y, más aún, cuando el número de grupos que tiene que atender un mismo docente también es alto. Pero que esté ya entre las preocupaciones de los estudiantes cuando inician su formación universitaria es significativo de su importancia para alcanzar aprendizajes de calidad.

Estos mismos estudiantes, cuando están a punto de finalizar su formación universitaria inicial como maestros, mantienen algunas percepciones similares a las que hacían en primer curso, pero su experiencia ha hecho que ahora expresen valoraciones diferentes. De este modo, la influencia de la enseñanza recibida y del ejemplo mostrado por sus docentes sigue considerándose como crucial en su formación y, a pesar de que destacan la formación recibida como modelo a imitar, son muchos los estudiantes que valoran que no ha sido la docencia esperada e, incluso, en ocasiones lo que se han encontrado es el ejemplo de aquello que no se debe realizar. Aunque no son las más numerosas, el hecho de que se encuentren varias respuestas aludiendo a las contradicciones entre la enseñanza recibida y la defendida por los docentes del Grado de Maestro en Educación Primaria debería ser una cuestión a atender para mejorar en un futuro. No se puede mantener una enseñanza transmisiva, individualista y centrada en la reproducción de información al estudiantado cuando se pretende formar docentes innovadores, con capacidad para trabajar cooperativamente y de adaptarse a alumnado diversos y situaciones complejas.

Si comparamos las innovaciones que esperaban encontrar cuando esta muestra de alumnos inició su carrera, con las que finalmente afirman haber vivido, podemos comprobar como las innovaciones tecnológicas han sido más llamativas o relevantes que las metodológicas (aunque éstas últimas siguen considerándose como las segundas más importantes vividas en su formación). Así, consideran que la principal diferencia con la formación recibida en Educación Secundaria son los recursos tecnológicos que han tenido para comunicarse con sus docentes o acceder a la información, pero del mismo modo valoran que en la mayoría de las ocasiones esto no ha supuesto grandes diferencias metodológicas en la forma de enseñar o plantearse su proceso de aprendizaje. 
Desde esta misma perspectiva, las innovaciones que mejor valoran son aquellas que les han permitido un rol de mayor protagonismo en su proceso de aprendizaje, al mismo tiempo que los han acercado a su futuro profesional como maestros. Y es en este aspecto, precisamente, en el que consideran que debería mejorarse la formación inicial universitaria que reciben como docentes, facilitándoles un mayor análisis de situaciones de clase, ejemplificando posibles recursos o estrategias a utilizar, adaptando los contenidos de las diferentes materias del Grado a aquellos que ellos deben enseñar en un futuro. Cuestiones que, sin duda, deben tenerse en cuenta para un planteamiento de mejora de la calidad de la enseñanza universitaria de esta carrera.

Por último, factores relacionados con las características que muestra el docente en la creación de ambientes de aprendizaje, tales como entusiasmo, motivación, dedicación, etc., se encuentran entre los aspectos que se destacan como necesarios para mejorar las experiencias formativas del alumnado, tal y como ya se hacía en primer curso y que parece no haberse encontrado de forma generalizada en estos cuatro años de carrera.

No podemos concluir sin resaltar la percepción de desilusión o decepción que algunos de los estudiantes de esta muestra declaran en relación con la docencia recibida, lo cual se convierte en acicate a seguir mejorando e investigando para modificar estas valoraciones.

\section{Referencias}

Bono, A. (2010) Los docentes como engranajes fundamentales en la promoción de la motivación de sus estudiantes. Revista Iberoamericana de Educación, 54(2), pp. 1-8.

Clark, B. (2011) Cambio sustentable en la universidad. Buenos Aires: U.P.

Hattie, J. (2003) Teachers make a difference: What is the research evidence? Invited address at the Australian Council for Educational Research conference, Melbourne.

Lafout, E. (1999) La convivencia como criterio de enseñanza en los colegios. Madrid: Universidad Autónoma de Madrid.

Muijs, D. y Reynolds, D. (2002) La enseñanza efectiva: evidencia y la práctica. London; Thousand Oaks: SAGE.

O’Connor, J. y Mc Dermott, I. (2008) Introducción al pensamiento sistémico. Barcelona: Urano.

Pérez del Viso de Palou, R. (2010) Motivación y didáctica innovadora en la formación de alumnado heterogéneo. En: Mainero y Mazzola (comps.) Los postgrados en Educación Superior en Argentina y Latinoamérica. San LUIS: ED. UNSLU y RAPES.

Rowe, K.J. (2004) In good hands? The importance of teacher quality. Educare News, 149, pp. 4-14.

Rodríguez, G., Gil, J. y García, E. (1996) Metodología de la investigación cualitativa. Málaga: Aljibe.

Ruiz, J. (2012) Metodología de la investigación cualitativa. Bilbao: Universidad de Deusto.

Siufi, B. (2008) La educación universitaria como I + C (inteligencia + creatividad). Ponencia presentada en el Simposio Autoorganizado: Presencias y Ausencias de la actitud creativa en la formación integral de los estudiantes universitarios. V Congreso Iberoamericano de Docencia Universitaria, celebrado en Valencia en octubre de 2008.

Tójar, J.C. (2006) Investigación cualitativa: comprender y actuar. Madrid: La Muralla.

Trillo, F. (2008) El escenario didáctico. En: F. Trillo, y L. Sanjurjo, Didáctica para profesores de a pie. Propuestas para comprender y mejorar la práctica (pp. 15-19). Rosario: Homo Sapiens. 